\title{
The Sensitivity of Power System Expansion Models on Climate Scenarios
}

\author{
Bruno U. Schyska ${ }^{1}$, Alex Kies ${ }^{2}$, Markus Schlott ${ }^{2}$, Lueder von Bremen ${ }^{1}$, Wided Medjroubi ${ }^{1}$ \\ ${ }^{1}$ German Aerospace Center (DLR), Institute of Networked Energy Systems, Oldenburg, Germany \\ ${ }^{2}$ Frankfurt Institute for Advanced Studies, Frankfurt, Germany
}

Power system expansion models are a widely used tool for planning power systems, especially considering the integration of renewable resources. Studies using these models form the basis for far-reaching political decisions. The backbone of power system models is an optimization problem, which depends on a number of economic and technical parameters. Although these parameters contain significant uncertainties, a consistent way to quantify the sensitivity to these uncertainties does not yet exist. Here, we analyze and quantify the sensitivity of a power system expansion model to the meteorological parameter time series based on a novel misallocation metric. We find that the sensitivity to the weather data is in the same order of magnitude as the sensitivity to the definition of cost. By comparing different climatic periods both from a meteorological perspective and with respect to the impacts on the optimal power system design we can, additionally, identify representative weather years and periods which should rather not be used for expansion planning. 